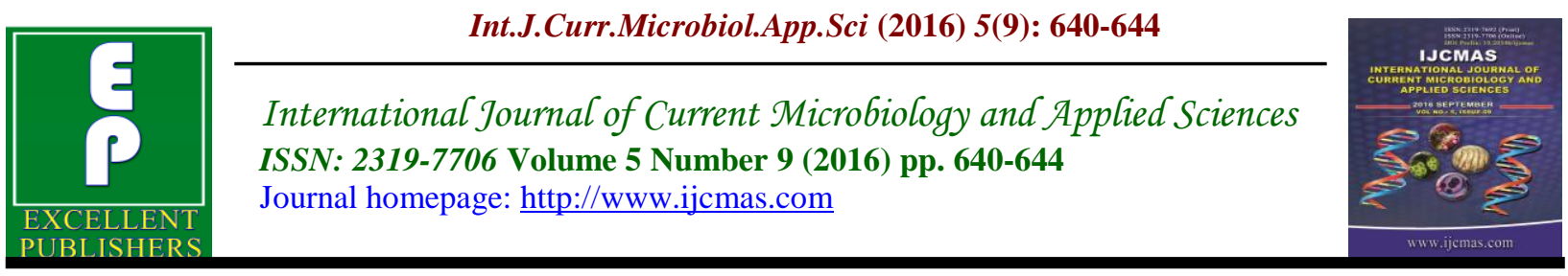

Original Research Article

http://dx.doi.org/10.20546/ijcmas.2016.509.073

\title{
Bacteriological Study of Catheter Associated Urinary Tract Infection in a Tertiary Care Hospital
}

\author{
G. Naveen*, C. Nagraj and G. Latha \\ Department of Microbiology, PES institute of Medical sciences \& Research \\ Kuppam - 517425, Chittoor, Andhra Pradesh, India \\ *Corresponding author
}

Keywords

CAUTI,

infection

control,

ESBL,

Escherichia coli.

Article Info

Accepted:

25 August 2016

Available Online:

10 September 2016
A B S T R A C T

Catheter associated urinary tract infections (CAUTI) are one of the most common cause of hospital acquired infections or nosocomial infections. Indwelling urinary catheterization in hospital setting has become a potential source for drug resistance and has now gained a prime importance in patient outcome. Hence, the aim of this study is to isolate uropathogens from CAUTI and find out antimicrobial sensitivity pattern among the isolates. This study was conducted over a duration of 2 years and urine samples were collected from 126 catheterized patients. The urine samples were processed microbiologically and antimicrobial sensitivity was performed. Out of 126 catheterized patients, 26 developed CAUTI and Escherichia coli 17 (65.3\%) was the most common organism followed by Proteus species $4(15.38 \%)$, Klebsiella pneumoniae 2 (7.6\%), Enterococcus species 2 $(7.6 \%)$ and staphylococcus epidermidis $1(3.8 \%)$. Enterobacteriaceae showed significant resistance to drugs like gentamycin, ciprofloxacin, and ceftazidime and were highly sensitive to drugs like imipenem, meropenem, nitrofurantoin, amikacin, ceftazidime - clavulinic acid and piperacillin - tazobactum. Among the gram positives, enterococcus $2(7.6 \%)$ was the most common organism followed by staphylococcus epidermidis $1(3.8 \%)$. Only one (3.8\%) ESBL producer was detected among gram negative bacilli. 26 patients $(20.63 \%)$ developed CAUTI among the analyzed 126 patients. Among these $13(50 \%)$ developed CAUTI on $7^{\text {th }}$ day of catheterization. Hence, dedicated infection control practice along with early diagnosis and timely therapeutic management is very important to control these CAUTI.

\section{Introduction}

Catheter associated urinary tract infection is one of the most common cause of hospital acquired infections. The indwelling catheterization has become a potential cause for morbidity among patients admitted in the hospital and has gained a prime importance in patient outcome. Bacteriuria develops in around 10 to $15 \%$ of hospitalized patients with indwelling urethral catheter. Among the community acquired pathogens $E$. coli is responsible for approximately 80 to $90 \%$ of cases followed by other gram negative bacilli and gram positive cocci. CAUTI in patients with indwelling urinary catheters is 
presumed by the presence of signs or symptoms compatible with urinary tract infection with no other identified source of infections along with $10^{5}$ or more $\mathrm{CFU} / \mathrm{ml}$ of one bacterial species in a single catheter urine specimen (48 hours catheterization) or in a mid stream voided urine specimen from a patient whose catheter has been removed within the previous 48 hours. In appropriate use of antimicrobial agents and inappropriate following of preventive bundles has led to increase in emergence of multidrug resistant uropathogens and increased risk of mortality in hospitalized patients (Hooton et al., 2009).

\section{Materials and Methods}

This study was conducted over a period of 2 years in a tertiary care hospital PESIMSR, Kuppam. A total of 126 urine samples were collected from patients who were catheterized.

The following patients were excluded from the study:

$>$ Patients who were earlier treated with UTI were excluded.

$>$ Patients already suffering from cystitis and prostatic enlargement.

$>$ Patients on suprapubic catheter, nephrostomy tube and condom catheter were excluded.

Samples were collected on first day of catheterization, later repeated every alternate day till $11^{\text {th }}$ day. First day sample was collected and processed to rule out prior urinary tract infection. The samples collected were inoculated onto blood agar and Macconkey agar by using standard calibrated loop. Samples which had $\geq 10^{5}$ $\mathrm{CFU} / \mathrm{ml}$ were processed further for biochemical reactions and antimicrobial sensitivity testing. Screening of ESBL producing strains was done using ceftriaxone, ceftazidime, aztreonam, cefotaxime and cefpodoxime. CAUTI in patients with indwelling urinary catheters is presumed by the presence of signs or symptoms compatible with urinary tract infection with no other identified source of infections along with $10^{5}$ or more $\mathrm{CFU} / \mathrm{ml}$ of one bacterial species in a single catheter urine specimen (48 hours catheterization) or in a mid stream voided urine specimen from a patient whose catheter has been removed within the previous 48 hours.

\section{Results and Discussion}

Out of the 126 patients analyzed, 26 (20.63\%) of them developed CAUTI. Among them 12 (46.1\%) were female and $14(53.9 \%)$ were male. $13(50 \%)$ of them developed CAUTI on the $7^{\text {th }}$ day of catheterization. Escherichia coli 17 (65.3\%) was the most common organism isolated followed by proteus species 4 (15.38\%), Klebsiella pneumoniae 2 (7.6\%), Enterococcus species 2 (7.6\%) and Staphylococcus epidermidis 1 (3.8\%). Gram negative bacilli were predominant than gram positive cocci. Among gram positive cocci Enterococcus species 2 (7.6\%) was the most common followed by Staphylococcus epidermidis 1 (3.8\%). The sensitivity of all the organisms was assessed based on CLSI guidelines. Enterobacteriaceae showed significant resistance to drugs like gentamycin, ciprofloxacin, and ceftazidime and were highly sensitive to drugs like imipenem, meropenem, nitrofurantoin, amikacin, ceftazidime - clavulinic acid and piperacillin - tazobactum. Among the gram positives, Enterococcus 2 (7.6\%) was the most common organism followed by Staphylococcus epidermidis 1 (3.8\%). Only one $(3.8 \%)$ ESBL producer (Klebsiella 
pneumoniae) was detected among gram negative bacilli.

Out of 126 catheterized patients analyzed for CAUTI, 26 (20.63\%) developed catheter associated urinary tract infection, which is similar to studies done by Kulkarni et al., (Kulkarni et al., 2014), Bhatia et al., (Bhatia et al., 2010), Bagchi et al., (Bagchi et al., 2015). Male predominance was seen compared to females, which was similar to that reported by Jaggi et al., (Namitha Jaggi et al., 2012). 23 (88.46\%) patients were found to develop CAUTI after 7 days of catheter insertion, which correlated with studies done by Kulkarni et al., Prolonged catheterization was found to be associated with increased cases of CAUTI, therefore proper maintenance and care of catheter is required to reduce the incidence of CAUTI (Sabir et al., 2014). Among the uropathogens isolated E. coli was the most common followed by Proteus vulgaris, Klebsiella, Enterococcus species and Staphylococcus epidermidis. These findings matched with that of Bagchi et al., Kazi et al., (Mohammad Mukhit Kazi et al., 2015), and Jayashri et al., (Jayashri et al., 2015).

Table.1 organisms isolated from urine of catheterized patients

\begin{tabular}{|c|c|c|}
\hline Organisms & Total isolates-n & Percentage \% \\
\hline Escherichia coli & 17 & $65.3 \%$ \\
\hline Klebsiella pneumoniae & 2 & $7.6 \%$ \\
\hline Proteus species & 4 & $15.38 \%$ \\
\hline Enterococcus species & 2 & $7.6 \%$ \\
\hline Staphylococcus epidermidis & 1 & $3.8 \%$ \\
\hline
\end{tabular}

Table.2 Antibiotic sensitivity pattern of gram positive organisms

\begin{tabular}{|c|c|c|}
\hline Antimicrobials & $\begin{array}{c}\text { Staphylococcus } \\
\text { epidermidis }(\%)\end{array}$ & $\begin{array}{c}\text { Enterococcus species } \\
(\%)\end{array}$ \\
\hline Ampicillin & 100 & 50 \\
\hline Amikacin & 100 & - \\
\hline Amoxycillin - clavulinic acid & 100 & - \\
\hline Clindamycin & 100 & - \\
\hline Co-trimoxazole & 100 & 50 \\
\hline Ciprofloxacin & 100 & 50 \\
\hline Erythromycin & 100 & - \\
\hline Gentamycin & 100 & - \\
\hline Levofloxacin & 100 & 00 \\
\hline Nitrofurantoin & 100 & 0 \\
\hline Norfloxacin & 100 & 0 \\
\hline Ofloxacin & 100 & 100 \\
\hline Penicillin & 0 & 100 \\
\hline Teicoplanin & 100 & \\
\hline Vancomycin & 100 & \\
\hline & & \\
\hline
\end{tabular}


Table.3 Antimicrobial sensitivity pattern of gram negative bacilli

\begin{tabular}{|c|c|c|c|}
\hline Antimicrobials & $\begin{array}{c}\text { Escherichia coli } \\
\mathbf{\%})\end{array}$ & $\begin{array}{c}\text { Klebsiella } \\
\text { pneumoniae } \\
(\boldsymbol{\%})\end{array}$ & $\begin{array}{c}\text { Proteus species } \\
(\%)\end{array}$ \\
\hline Amikacin & 87 & 100 & 75 \\
\hline Ampicillin - sulbactum & 87 & 50 & 75 \\
\hline Ceftriaxone & 72 & 50 & 50 \\
\hline Ceftazidime & 74 & 50 & 75 \\
\hline Ceftazidime - clavulinic acid & 88 & 50 & 100 \\
\hline Ciprofloxacin & 24 & 50 & 25 \\
\hline Gentamycin & 87 & 50 & 25 \\
\hline Imipenem & 100 & 100 & 100 \\
\hline Meropenem & 100 & 100 & 100 \\
\hline Nitrofurantoin & 94 & 100 & 100 \\
\hline Piperacillin - tazobactum & 87 & 50 & 75 \\
\hline
\end{tabular}

Enterobacteriacae showed significant resistant to common antibiotics like Ciprofloxacin, gentamycin, and ceftriaxone. Most of the gram negative bacilli showed complete sensitivity to drugs like nitrofurantoin, imipenem, meropenem, piperacillin-tazobactum, and ampicillinsulbactum. Most of the gram positives showed complete sensitivity to drugs like vancomycin, teicoplanin, and nitrofurantoin (Anupurba et al., 2003).

In conclusion, prolonged catheterization is an important risk factor for the development of hospital acquired infections like CAUTI. Among the uropathogens isolated E. coli is the most common agent. Uropathogens isolated from CAUTI showed one ESBL producer and some of them resistant to commonly used antibiotics. This shows that appropriate timely diagnosis and management is very important to control these pathogens. Strict aseptic precaution has to be taken while insertion and daily catheter care to reduce CAUTI. Strict antibiotic stewardship and antibiotic policy following can reduce inappropriate use of antimicrobials.

\section{Acknowledgement:}

We are thankful to the whole infection control team, intensivist and quality team (QMS) of PESIMSR, Kuppam in bringing out this study.

\section{References}

Anupurba, S., Sen, M.R., Nath, G., Sharma, B.M., Gulati, A.K., Mohapatra, T.M. 2003. Prevalence of methicillin resistant Staphylococcus aureus in a tertiary referral hospital in eastern Uttar Pradesh. Indian J. Med. Microbiol., 21(1): 4951.

Bagchi, I., Jaitly, N.K., Thombare, V.R. 2015. Microbiological Evaluation of Catheter Associated Urinary Tract Infection in a Tertiary Care Hospital. PJSR, 8(2): 23-29.

Bhatia, N., Daga, M.K., Garg, S., Prakash, S.K. Urinary catheterization in medical wards. J. Glob. Infect. Dis., 2(2): 83-90.

Hooton, T.M., Bradley, S.F., Cardenas, D.D., Colgan, R. et al. 2009. Diagnosis, Prevention, and Treatment of CAUTI in Adults: 2009 
International Clinical Practice Guidelines from the Infectious Diseases Society of America.

Jayshri, V., Dund, Rakesh Ninama, Mala Sinha. 2015. Antibiotic Sensitivity Pattern of Bacteria Isolated from Catheter Associated Urinary Tract Infections in Tertiary Care Hospital, Jamangar, Sch. J. App. Med. Sci., 3(5C): 1985-1988.

Kulkarni, S.G., S.H. Talib, Manjiri Naik, Amit Kale. 2014. Profile of Urinary Tract Infection in Indwelling Catheterized, (IOSR-JDMS) e-ISSN: 2279-0853, p-ISSN: 2279-0861. Volume 13, Issue 4 Ver. IV. (Apr. 2014), PP 132-138.

Mohammad Mukhit Kazi, Amol Harshe, Hanumant Sale, Dileep Mane, Minal Yande and Supriya Chabukswar. 2015. Catheter Associated Urinary
Tract Infections (CAUTI) and Antibiotic Sensitivity Pattern from Confirmed Cases of CAUTI in a Tertiary Care Hospital: A Prospective Study, Clin. Microbiol., 4: 2.

Namita Jaggi and Pushpa Sissodia. Multi dimentional supervision programme to reduce CAUTI and its analysis to enable focus on labour cost effective infection control in a tertiary care hospital. J. Clin. Diagn. Res., 6(8): 1372-1376.

Sabir, S., Ahmad Anjum, A., Ijaz, T., Asad Ali, M., Ur Rehman Khan, M., et al. 2014. Isolation and antibiotic susceptibility of $E$. coli from urinary tract infection in a tertiary care hospital, Pak. J. Med. Sci., 30: 389392.

\section{How to cite this article:}

Naveen, G., C. Nagraj and Latha, G. 2016. Bacteriological Study of Catheter Associated Urinary Tract Infection in a Tertiary Care Hospital. Int.J.Curr.Microbiol.App.Sci. 5(9): 640644. doi: http://dx.doi.org/10.20546/ijcmas.2016.509.073 